University of Wollongong

Research Online

Faculty of Social Sciences - Papers (Archive) Faculty of Arts, Social Sciences \& Humanities

2013

Varying task difficulty in the Go/Nogo task: The effects of inhibitory control, arousal, and perceived effort on ERP components

Nicholas Benikos

University of Wollongong, npb95@uowmail.edu.au

Stuart J. Johnstone

University of Wollongong, sjohnsto@uow.edu.au

Steven J. Roodenrys

University of Wollongong, steven@uow.edu.au

Follow this and additional works at: https://ro.uow.edu.au/sspapers

Part of the Education Commons, and the Social and Behavioral Sciences Commons

Research Online is the open access institutional repository for the University of Wollongong. For further information contact the UOW Library: research-pubs@uow.edu.au 


\title{
Varying task difficulty in the Go/Nogo task: The effects of inhibitory control, arousal, and perceived effort on ERP components
}

\begin{abstract}
Similar to other executive functions, inhibitory control is thought to be a dynamic process that can be influenced by variations in task difficulty. However, little is known about how different task parameters alter inhibitory performance and processing as a task becomes more difficult. The aim of this study was to investigate the influence of varying task difficulty, via manipulation of reaction time deadline (RTD), on measures of inhibitory control, perceived effort, and task-related arousal (indexed by skin conductance level). Sixty adults completed a visual Go/Nogo task $(70 \% \mathrm{Go})$ after being randomly assigned to one of three task difficulty conditions: High, Medium and Low, with RTDs of 300, 500 or 1000 ms, respectively. Results revealed incremental increases in Go/Nogo errors and greater perceived effort with increasing difficulty. No condition differences were found for arousal, but the amplitude of the Nogo N2 increased and peaked earlier with increasing task difficulty. In contrast, the Nogo P3 effect was reduced in the High condition compared to the Low and Medium conditions. Finally, the amplitude of N1 and P2 showed differential effects, with Nogo N1 increasing with task difficulty, while the Nogo P2 decreased. This study provides valuable baseline behavioural and ERP data for appropriately manipulating difficulty (via RTD) in Go/Nogo tasks - highlighting the potential key role of not only the N2 and P3, but also the N1 and P2 components for task performance.
\end{abstract}

\section{Keywords}

components, task, effort, perceived, arousal, erp, control, varying, inhibitory, effects, nogo, go, difficulty

\section{Disciplines}

Education | Social and Behavioral Sciences

\section{Publication Details}

Benikos, N., Johnstone, S. J. \& Roodenrys, S. J. (2013). Varying task difficulty in the Go/Nogo task: The effects of inhibitory control, arousal, and perceived effort on ERP components. International Journal of Psychophysiology, 87 (3), 262-272. 


\section{Varying task difficulty in the Go/Nogo task: the effects of inhibitory control, arousal, and perceived effort on ERP components}

Nicholas Benikos

University of Wollongong, npb95@uow.edu.au

Stuart J. Johnstone

University of Wollongong, stuart_johnstone@uow.edu.au

Steven J. Roodenrys

University of Wollongong, steven@uow.edu.au

Publication details: Benikos, N., Johnstone, S. J. \& Roodenrys, S. J. (2013). Varying task difficulty in the Go/Nogo task: The effects of inhibitory control, arousal, and perceived effort on ERP components. International Journal of Psychophysiology, 87 (3), 262-272. 


\begin{abstract}
Similar to other executive functions, inhibitory control is thought to be a dynamic process that can be influenced by variations in task difficulty. However, little is known about how different task parameters alter inhibitory performance and processing as a task becomes more difficult. The aim of this study was to investigate the influence of varying task difficulty, via manipulation of reaction time deadline (RTD), on measures of inhibitory control, perceived effort, and task-related arousal (indexed by Skin Conductance Level). Sixty adults completed a visual Go/Nogo task ( $70 \% \mathrm{Go})$ after being randomly assigned to one of three task difficulty conditions: High, Medium and Low, with RTDs of 300, 500 or $1000 \mathrm{~ms}$, respectively. Results revealed incremental increases in Go/Nogo errors and greater perceived effort with increasing difficulty. No condition differences were found for arousal, but the amplitude of the Nogo N2 increased and peaked earlier with increasing task difficulty. In contrast, the Nogo P3 effect was reduced in the High condition compared to the Low and Medium conditions. Finally, the amplitude of N1 and P2 showed differential effects, with Nogo N1 increasing with task difficulty, while the Nogo P2 decreased. This study provides valuable baseline behavioural and ERP data for appropriately manipulating difficulty (via RTD) in Go/Nogo tasks - highlighting the potentially key role of not only the $\mathrm{N} 2$ and $\mathrm{P} 3$, but also the N1 and P2 components for task performance.
\end{abstract}

\title{
Keywords:
}

Task difficulty; Inhibition; N2; P3; Arousal; Effort; ERPs; time pressure 


\section{Introduction}

Inhibitory control refers to the ability to successfully suppress thoughts, behaviour and irrelevant stimuli (Aron et al., 2004). Crucial for the proper functioning of many other cognitive capacities (Clark, 1996), inhibitory control is an important, but often unnoticed, feature of everyday life: Its effective execution potentially means the difference between safely crossing a busy road or endangering oneself to oncoming traffic.

Among the most commonly employed paradigms used to investigate inhibitory processing is the $\mathrm{Go} / \mathrm{Nogo}$ task, which requires participants to respond to a frequently presented Go stimulus, while withholding a response to a rare Nogo stimulus. Event-related potentials (ERPs) to Go/Nogo tasks typically contain two inhibition-related components: an augmented N2 for Nogo relative to Go stimuli, primarily at frontal sites (e.g. Falkenstein et al., 1999; Fallgatter and Strik, 1991; Oddy et al., 2005), and a more anterior focus for the Nogo P3, where P3 is larger for Nogo than Go stimuli at frontal and central leads. The Nogo N2 has been suggested to reflect the pre-motor 'need' for inhibition (Kok, 1986), but more recent research has instead linked the N2 to response conflict (Donkers and van Boxtel, 2004; Nieuwenhuis et al., 2003). By contrast, the Nogo P3 has primarily been related to motor inhibition in recent years (Smith, Johnstone \& Barry, 2006, 2007, 2008; Smith et al., 2010). But further work has also suggested that it may not be linked to inhibition itself, but more to the evaluation of the inhibitory process (Band and van Boxtel, 1999; Bruin et al., 2001). Notably, both components appear to be modulated by different neurobiological pathways (Beste et al., 2008; Beste et al., 2010) supporting the idea that they reflect different inhibition-related sub-processes.

Like other executive functions, inhibitory control is assumed to be a dynamic process that should be influenced by variations in task difficulty. However, relatively little is known about how different experimental parameters affect the behavioural and neural underpinnings of this ability (Beste et al., 2010; Lindqvist and Thorell, 2009; Thorell et al., 2009). There are a number of key reasons why it is important to study the influence of task difficulty on inhibitory control. Firstly, from a clinical perspective, the nature of inhibition deficits can only be ascertained if the paradigms employed are sufficiently difficult to differentiate performance between clinical subjects and healthy controls (Beste et al., 2010; 
Lindqvist and Thorell, 2009). Further, variations in task difficulty, in and of themselves, have been linked to differences in neural activation, leading to inconsistencies in the Go/Nogo literature (for a meta-analysis see Simmonds et al., 2008). Baseline ERP data are required to clarify these effects. Finally, the possibility of developing targeted inhibition training paradigms as an adjunct to existing rehabilitation programs may offer a potentially useful aid for individuals suffering from deficits in inhibitory control (for e.g. Attentiondeficit/Hyperactivity disorder, ADHD; Johnstone et al., 2010; Thorell et al., 2009). Training outcomes in these studies may be enhanced if the approach taken is based on fundamental research into the optimal way to manipulate inhibition difficulty. Thus, studying how task difficulty influences inhibitory control is important from both a 'pure science' and applied perspective, and is the major aim of this study.

Previous research examining the influence of task difficulty on inhibition-related ERP components has been varied with respect to methodologies and findings. Jodo and Kayama (1992) manipulated task difficulty with reaction time deadline, asking one group of participants to respond within $300 \mathrm{~ms}$ of the Go signal, and another to respond within 500 ms. They reported an enhancement of the Nogo N2 only in the fast responders. Although this effect was interpreted as being due to increased inhibition difficulty, this was unable to be confirmed since no behavioural results for inhibitory performance were reported. In a subsequent investigation, Band, Ridderinkhof and van der Molen (2003) divided participants into one of two instructional conditions: a speed condition, where subjects were required to respond as fast as possible, and a balance condition, where speed as well as accuracy was emphasised. The speed of response was found to modulate both inhibitory performance and ERPs, with increased Nogo errors and Nogo N2 for the speed condition. In contrast to these reports, Smith et al. (2006), who separated participants into 'fast' and 'slow' responders via median split post-hoc, reported no differences for the N2.

Furthermore, despite clear effects being reported for the N2, the Go/Nogo literature examining the influence of task difficulty on the P3 is limited. Previous investigations have either not considered the P3 (Band et al., 2003; Jodo and Kayama, 1992), or have used a 50/50 Go/Nogo split (Jodo and Kayama, 1992; Smith et al., 2006) which may not reliably induce prepotent response inhibition, depending on the paradigm (e.g. Braver et al., 2001; Tekok-Kilic et al., 2001). Moreover, these studies have generally only employed two difficulty levels (i.e. low vs. high). Given that both theoretical viewpoints (e.g. Cognitiveenergetic model; Sanders, 1983) and experimental findings (Wodka et al., 2009) have 
suggested performance improvements only during moderate rather than easy/hard difficulty levels, the use of the three task difficulty conditions in the present study allows examination of a range of effects, rather than simply assuming linear changes. Thus, one aim of this study was to extend previous research by clarifying the effect of task difficulty (as manipulated by reaction time deadline: RTD) on not only the $\mathrm{N} 2$, but also the $\mathrm{P} 3$, using a 70/30 Go/Nogo split and three difficulty conditions (Low, Medium and High).

Although the main focus of this study was the influence of task difficulty on inhibitory processing, the measurement of skin conductance level (SCL) - a well-established measure of central nervous system (CNS) arousal (Barry and Sokolov, 1993) - allows examination of the effect of arousal level on inhibitory performance and processing. A review of the literature suggests that arousal may amplify or improve task performance (for a discussion see VaezMousavi et al., 2007), which may be characterised by an inverted-U relationship, where moderate levels of physiological arousal result in optimal performance, with a deterioration in performance seen during low-or high-arousal levels (Yerkes and Dodson, 1908). Additionally, as initially proposed by Yerkes and Dodson (1908), optimal arousal levels may depend on the difficulty of a given task. In line with the findings of Yerkes and Dodson (1908) are results showing that inhibition performance was optimised only at moderate inter-stimulus intervals (ISIs;Wodka et al., 2009). Further work by Barry et al. (2007) has reported that increased arousal, via caffeine ingestion, resulted in not only increased SCL, but also concurrent improvements in Go/Nogo performance. However, findings from research using similar tasks have been mixed, showing no relationship between arousal and performance (Barry et al., 2005; VaezMousavi et al., 2009; VaezMousavi et al., 2007). The paucity of errors in the previous studies may help to explain these results, and as such, the manipulation of task difficulty would ensure greater errors and help to more thoroughly explore the arousal/performance link.

In sum, this study sought to extend previous research by examining the behavioural and neural effects of varying task difficulty, via RTD, on inhibitory processing. To this end, we used a modified version of the Go/Nogo task that required the inhibition of a prepotent response during three task difficulty conditions: Low (1000 ms), Medium (500 ms) and High (300 ms). As mentioned above, the Nogo N2 and Nogo P3 have been associated with different aspects of response inhibition so the ERP analyses focused on these components. While no specific predictions were made for the early ERP components, given the potential modulatory effects of task difficulty on early stimulus processing (e.g. Miller et al., 2011), 
any differences found would be explored. Moreover, participants provided perceived effort ratings and we recorded skin conductance to assess the contribution of arousal on performance and processing.

\section{Method}

\subsection{Participants}

A total of 69 adults enrolled in the present study to fulfil an undergraduate course requirement, with three being excluded according to the selection criteria. To be included in the study, participants were required to refrain from caffeine for 2 hours prior to testing and have not taken any psychotropic substances (prescription or illegal) for 24 hours prior to testing, or no more than once a month in the previous six months. Participants were also screened for neurological disorders and all reported normal or corrected-to-normal vision.

The remaining 66 participants were randomly assigned to one of three conditions: Low, Medium or High task difficulty. Of these, data from 6 subjects were rejected either due to excessive eye artefact (3 participants), technical problems ( 2 participants) or for failure to perform the task properly ( 1 participant). Therefore, 20 participants each were included in the final analyses for the Low (Low: 17 females, 3 males, mean age 21.23, SD 4.12), Medium (14 females, 6 males, mean age 21.5, SD 5.89) and High condition (14 females, 6 males, mean age 21.4, SD 3.32). All but 5 of the 60 participants were right-handed. The research protocol was approved by the joint University of Wollongong and Illawarra Area Heath Service Human Research Ethics Committee.

\subsection{Task}

Stimuli were generated using Presentation (Version 11.0; Neurobehavioral Systems, Albany, CA, USA). Each trial began with a central fixation cross (+) presented for a variable interval of $500-1000 \mathrm{~ms}(\mathrm{M}=750 \mathrm{~ms})$, followed by the Go/Nogo stimulus presented in the centre of 
the screen for $200 \mathrm{~ms}$. A blank screen then replaced the stimulus for a variable blank period of $1250-1750 \mathrm{~ms}(\mathrm{M}=1250 \mathrm{~ms})$. Within this period, participants in the High, Medium and Low task difficulty conditions were required to respond via a button press to Go stimuli within 300,500 or $1000 \mathrm{~ms}$, respectively (see Figure 1), or to refrain from responding to Nogo stimuli. Performance feedback was provided via the following fixation cross, which remained white for correct response, but changed to a red colour for incorrect responses. Incorrect responses (i.e. presses to Nogo stimuli during the variable blank period, omissions and responses outside the RTD) were recorded in order to calculate error rates. Only presses to the Go stimulus within the predefined response window were regarded as correct.

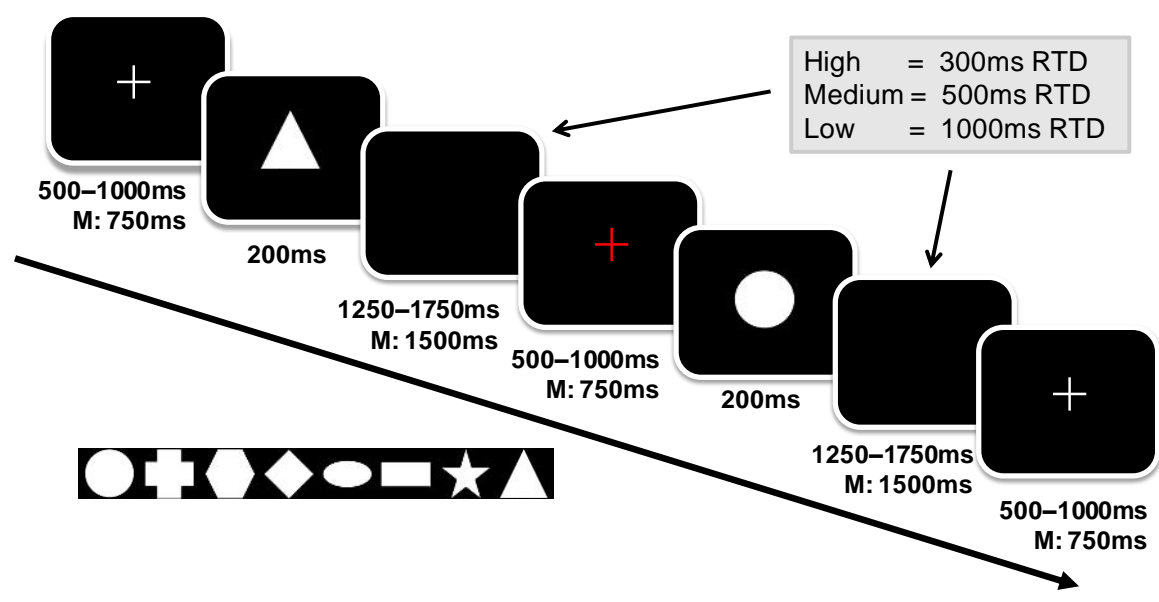

Figure 1. Schematic presentation of each task difficulty condition to Go (triangle) and Nogo (circle) stimuli.

After an initial practice block of 30 trials (50\% Nogo), all participants completed eight experimental blocks (30\% Nogo) of 100 trials each. Only data from the first two blocks is reported here. Target Go/Nogo stimuli for each block was selected from a pool of eight shapes (i.e. triangle, cross, hexagon, diamond, ellipse, rectangle, star and circle; see Figure 1) and were presented on a 15 " computer monitor, with participants seated one metre from the screen. The stimuli measured approximately $3 \times 3 \mathrm{~cm}$ on the screen. Presentation of shape stimuli were counterbalanced using a Latin square design (Bradley, 1958), with Go/Nogo response assignment counterbalanced across subjects. Total task time was approximately 43 minutes. 


\subsection{Procedure}

Participants were given an outline of the testing procedure and familiarised with the laboratory equipment before informed consent was given. The experimenter emphasised that participants could withdraw at any time without penalty. They were then asked to complete a short screening questionnaire to assess vision problems, medication/psychotropic substance use, and neurological disorders. Subjects were then fitted with EEG and skin conductance recording equipment, and seated in a dimly-lit sound-attenuated and electrically-shielded testing booth. An incandescent light in the booth was dimmed for the duration of the experiment. An initial 3 min baseline recording was conducted while participants were asked to sit quietly with eyes closed. Subjects were then presented with a modified Go/Nogo task and were instructed that they would see either of two shapes, one representing the Go stimulus, and the other representing the Nogo stimulus. They were asked to press the button before the pre-determined RTD with the thumb of their right hand to Go stimuli, and to refrain from responding to Nogo stimuli. Performance feedback was provided by the following fixation cross, which changed from a white to red colour on incorrect trials (i.e. Go responses exceeding the RTD and presses to Nogo stimuli) and remained white on correct trials. Participants were asked to "do their best" to avoid the incorrect feedback, and were encouraged to keep as still as possible and to minimise eye movements during the testing blocks. Go/Nogo shape assignment was shown on the screen and verbally confirmed by the participant prior to each block. After a short practice block, all participants completed the experimental blocks. At the end of each block, mean Go RT, the percentage of Go and Nogo errors were displayed for subjects to review. They were then asked to rate their perceived level of effort with the question "How much effort did you use to complete that block?" and responded by a 5-point Likert scale ranging from: $1=$ Very little, $2=$ Moderate effort, and 5 = Everything I had. Prior to the first rating a basic example was shown to the subject to ensure understanding. Participants were given a short break at the end of each block and asked to continue on.

\subsection{Electrophysiological recording}

The continuous scalp electroencephalogram (EEG was recorded from 19 sites (Fp1, Fp2, F3, F4, F7, F8, Fz, C3, C4, Cz, P3, P4, Pz, T3, T4, T5, T6, O1, O2) using an electrode cap containing tin electrodes fitted according to the international 10-20 system (Jasper, 1958). A 
ground electrode located between Fpz and Fz, and all electrodes were referenced to linked ears. EOG was measured vertically with two tin cup electrodes, $1 \mathrm{~cm}$ above and below the left eye. Impedance was kept below $3 \mathrm{k} \Omega$ for EOG and reference electrodes, and below $5 \mathrm{k} \Omega$ for cap electrodes. EEG and EOG signals were amplified 19 times and sampled at $500 \mathrm{~Hz}$, with bandpass down $3 \mathrm{db}$ at 0.1 and $100 \mathrm{~Hz}$ via a NuAmps system (Compumedics Limited, Melbourne, Australia). Prior to processing, the EEG data were digitally filtered using a lowpass filter $3 \mathrm{db}$ down at $30 \mathrm{~Hz}$.

\subsection{Skin Conductance recording}

Electrodermal activity was recorded using two $\mathrm{Ag} / \mathrm{AgCl}$ electrodes placed on the distal phalanges of the third and fourth digits of the left hand. Recording electrodes were filled with electrode paste $(0.05 \mathrm{M} \mathrm{NaCl}$ in an inert viscous ointment base) and secured using velcro straps and tape. A constant voltage device (UFI Bioderm model 2701) set at $0.5 \mathrm{~V}$ was used. This system separately recorded tonic DC-coupled SCL and AC-coupled skin conductance fluctuations (Skin Conductance Response; SCR), measured in microsiemens $(\mu \mathrm{S})$. Only SCL is reported here.

\subsection{Data Quantification}

The ERP epoch was defined as $100 \mathrm{~ms}$ pre-stimulus to $900 \mathrm{~ms}$ post-stimulus onset. Epochs were excluded if they contained activity greater than $\pm 100 \mu \mathrm{V}$ at any non-frontal site. EOG artefact reduction was carried out based on vertical EOG (Semlitsch et al., 1986). ERPs were averaged across epochs for correct responses only. This resulted in a minimum of 32 artefact-and-error-free Nogo trials being included in each average. Go epochs were averaged separately, chosen randomly from the available correct Go epochs to equal the number of Nogo epochs. Grand average ERP waveforms for Go and Nogo stimuli were displayed in order to define the components latency range. Latency was fixed across sites to the peak latency of the site of maximum amplitude (Picton et al., 2000; Spencer et al., 2001). ERP component peaks were quantified using automatic peak-picking software which identified the largest positive or negative deflections within the predefined latency range, relative to the $100 \mathrm{~ms}$ pre-stimulus baseline period. Peak latency ranges and sites were as follows: N1 
(100 -160 ms Fz), P2 (180-240 ms Pz), N2 (200-280 ms Fz), P3 (280-520 ms Pz). Skin

conductance level was taken as the average value (in $\mu \mathrm{S}$ ) for each $30 \mathrm{sec}$ period over the 3.5 min duration of each block of the Go/Nogo task.

\subsection{Statistical analyses}

The error rate (Go omission errors, RTD and Nogo errors) were calculated as the number of responses divided by the total number of presentations. Univariate analysis of variance (ANOVA) was used to analyse task performance perceived effort and skin conductance level data with Condition (Low vs. Medium vs. High) as the between-subjects factor. Planned polynomial (Linear, Quadratic) contrasts were used to analyse differences within Condition.

Primary analyses of the ERP data were restricted to the sites F3, Fz, F4, C3, Cz, C4, P3, Pz and P4. Go and Nogo data were subject to a Condition [Low (L) vs. Medium (M) vs. High (H)] x Lateral (Left vs. Midline vs. Right) x Sagittal (Frontal vs. Central vs. Parietal) x Stimulus (Go vs. Nogo) ANOVA, with repeated measures on the within-subjects factors. Differences within Condition were assessed using polynomial contrasts (Linear, Quadratic). Analyses for component latency omitted the site contrasts. Planned orthogonal contrasts, which allow insight into the topographic distribution of each component, were performed on the within-subjects factors. The Lateral factor compared activity in the left hemisphere (mean of F3, C3 and P3) with the right (mean of F4, C4 and P4), and the mean of these with activity in the midline region (mean of $\mathrm{Fz}, \mathrm{Cz}$ and $\mathrm{Pz}$ ). Contrasts within the Sagittal factor compared frontal activity (mean of F3, Fz and F4) with parietal (mean of P3, Pz and P4), and the mean of these with activity in the central region (mean of $\mathrm{C} 3, \mathrm{Cz}$ and $\mathrm{C} 4$ ). As these contrasts were planned with no more of them than the degrees of freedom for each effect, no Bonferroni type adjustment to $\alpha$ were necessary (Tabachnick and Fidell, 1996). Also, single degrees of freedom contrasts are not affected by violations of symmetry assumptions common in repeated measures analyses, and thus do not require Greenhouse-Geisser-type corrections. As these analyses are carried out over a substantial number of variables, each may be considered to constitute a separate experiment. It should be noted that this increases the frequency of type 1 errors, however, as this is an increase in frequency, rather than probability, it cannot be 'controlled' by adjustment of a levels (Howell, 2009). All ERP statistics have $(1,58)$ degrees of freedom unless otherwise indicated. Outliers in the data 
were corrected for by replacing with the series mean. Data were normalised using the vector scaling method (McCarthy and Wood, 1985), and only interactions with topography that remained significant in the normalised data are reported here.

\section{Results}

\subsection{Manipulation check and perceived effort}

As can be seen in Figure 2, participants perceived effort was greater in the High than Medium and Low conditions (Linear: $F=6.64, p=.013, \eta 2=.104$ ), suggesting that the difficulty manipulation was successful, with greater perceived effort seen with each increase in task difficulty.

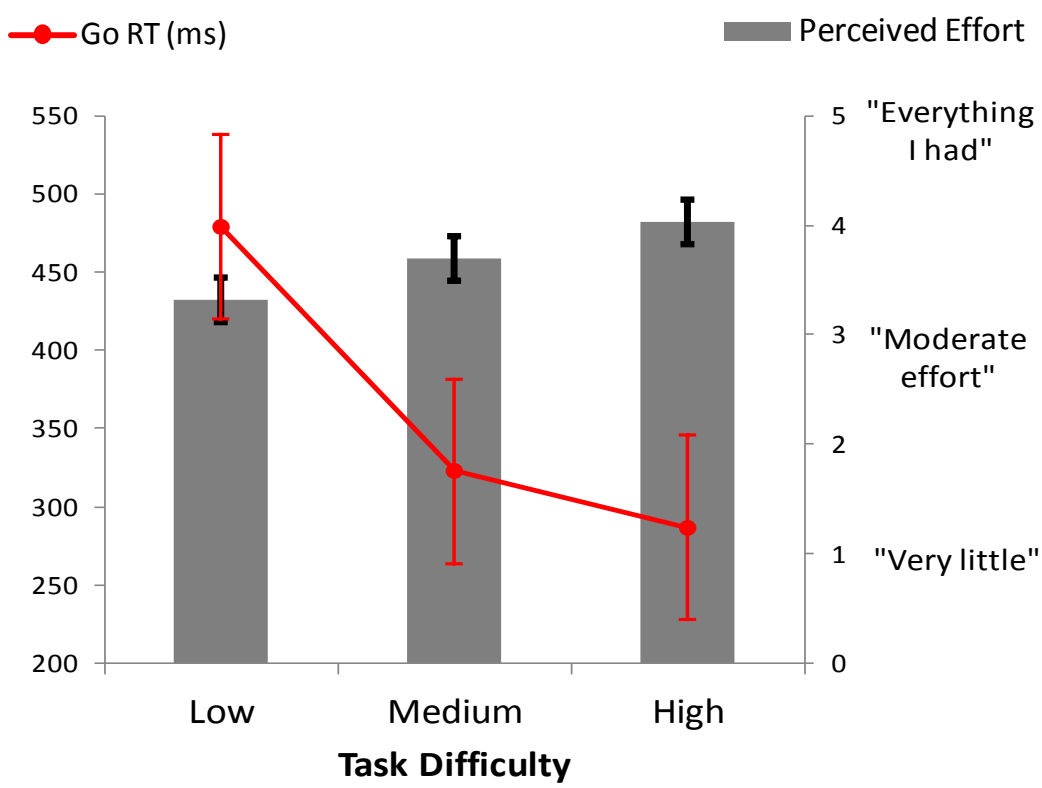

Figure 2. Reaction time and perceived effort ratings for each task difficulty condition. Error bars represent standard error of the mean. 


\subsection{Task performance}

Means and standard deviations of RT and errors are summarised in Table 1. Consistent with our experimental manipulation, RT to Go stimuli decreased with each RTD reduction (L > $\mathrm{M}>\mathrm{H}$; Linear: $F=403.55, p<.001, \eta 2=.787)$, with the steepest drop from the Low to Medium conditions (Quad: $F=52.02, p<.001, \eta 2=.101$ ).

Both Go RTD and omission errors (Go Om) showed linear (Go RTD: $F=222.93, p<.001$, $\eta 2=.673$; Go Om: $F=38.80, p<.001, \eta 2=.382$ ), and quadratic trends (Go RTD: $F=$ 51.31, $p<.001, \eta 2=.155$; Go Om: $F=4.17, p=.046, \eta 2=.043$ ), highlighting a steep increase in Go errors with increasing task difficulty, particularly apparent for the High condition. Inhibition performance showed a similar pattern, with incremental increases in Nogo errors with increasing task difficulty (i.e. $\mathrm{H}>\mathrm{M}>\mathrm{L}$ ), with the greatest percentage of errors seen in the High condition (Linear: $F=45.62, p<.001, \eta 2=.423$; Quad: $F=5.15, p$ $=.027, \eta 2=.048)$.

Table 1.

Summary statistics for task performance measures for each task difficulty condition.

\begin{tabular}{lrrr}
\hline & \multicolumn{3}{c}{ Task Difficulty } \\
\cline { 2 - 4 } & Low & Medium & High \\
\hline$R T(m s)$ & & & \\
Go RT & 479.0 & 323.0 & 286.6 \\
SDRT & 87.4 & 67.4 & 57.7 \\
& & & \\
Error rate (\%) & & & \\
Go RTD & 0.0 & 2.7 & 30.9 \\
Go Omission & 0.7 & 1.6 & 5.0 \\
Nogo Errors & 7.4 & 11.1 & 25.0 \\
\hline
\end{tabular}




\subsection{Skin conductance level}

While SCL appeared to show a quadratic trend among task difficulty conditions (i.e. H/L > M), no significant differences were found between the High (12.49 $\mu \mathrm{S})$, Medium (10.80 $\mu \mathrm{S})$ or Low conditions (12.17 $\mu$ S; Quad: $F=1.90, p=.174)$.

\subsection{Event related potentials}

Figure 3 presents grand mean ERPs to Go and Nogo stimuli across groups (top left panel) and for each condition separately (remaining three panels), with scalp distribution maps for each component in Figure 4. The waveforms are characterised by an N1-P2 complex, most apparent at frontal and central sites. An N2 component is apparent at about 270 ms primarily in the frontocentral region. Evident at approximately $300-400 \mathrm{~ms}$ post-stimulus, the $\mathrm{P} 3$ is a large positivity which peaks parietally for the Go condition and central-frontally for the Nogo condition. 

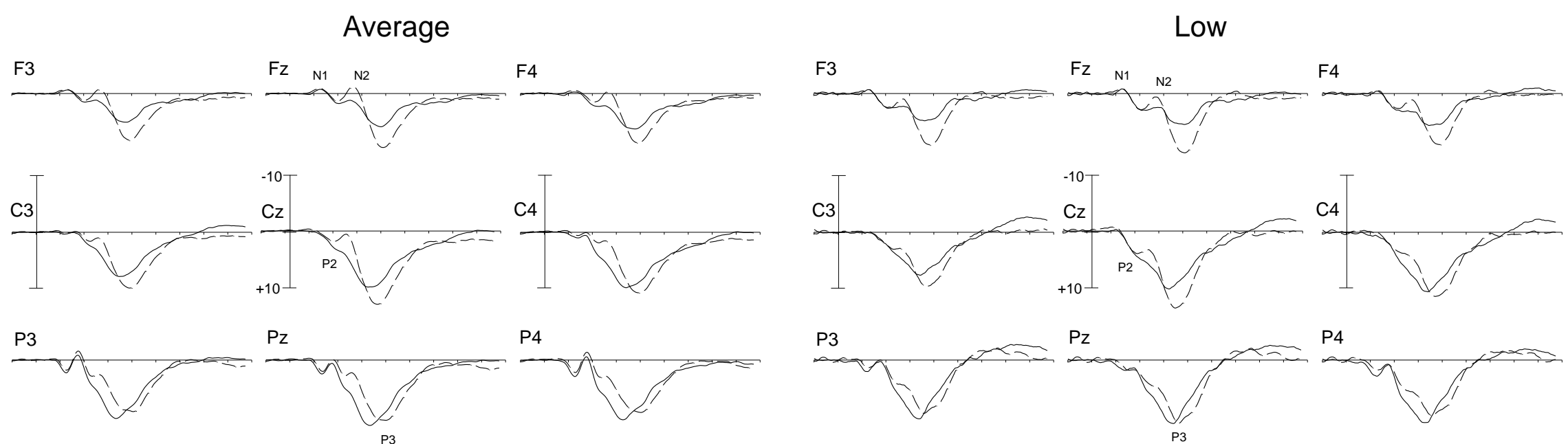

\section{Medium}

F3
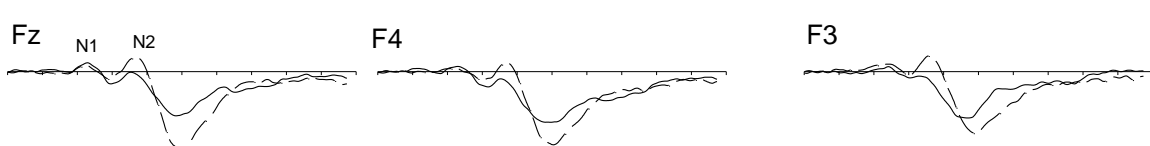

High
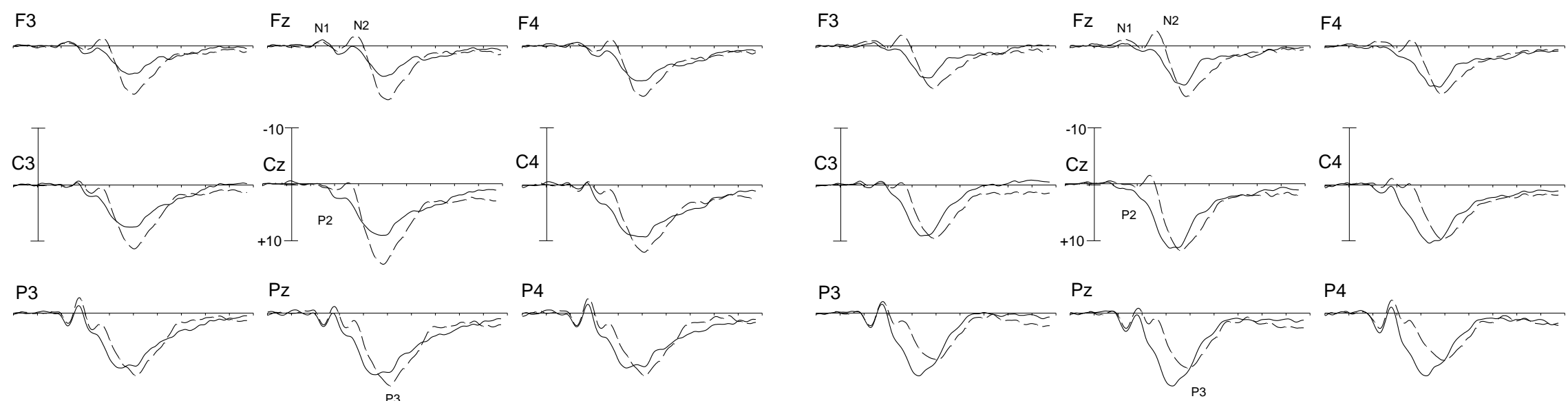

Figure 3. Grand mean ERPs to Go (solid line) and Nogo (dashed line) across condition (top left panel) and for each task difficulty condition separately (remaining three panels) at nine scalp locations. 


\subsubsection{N1}

N1 peaked at $143.7 \mathrm{~ms}$, with no condition differences for latency (Low $=138.9 \mathrm{~ms}$, Medium $=144.5 \mathrm{~ms}$, High $=147.7 \mathrm{~ms}$ ).

The general topography of the N1 (i.e. across stimulus and condition) showed a frontocentral maximum, with a left-midline focus (see Table 2 for effect summaries and means). Between task difficulty conditions and across stimulus, the central > frontoparietal difference was reduced with increasing task difficulty (i.e. $\mathrm{L}>\mathrm{M}>\mathrm{H}$ ), highlighting a larger $\mathrm{N} 1$ amplitude in posterior regions for the Medium/High, relative to the Low condition. On the Lateral dimension, the Low condition showed a large midline > hemispheres effect, in contrast to the Medium and High conditions, which displayed little hemispheric variation.

Notably, there was a significant difference for the N1 to Go vs. Nogo stimuli among the conditions. The Low condition showed a clear Go > Nogo N1, while this effect was reduced to be almost equipotential for the Medium condition, and reversed for the High condition (i.e. Nogo > Go N1; see Figure 4 for head maps and Figure 5, top left panel, for Go vs. Nogo comparisons).

\subsubsection{P2}

P2 peaked at $226.1 \mathrm{~ms}$, with no condition differences in latency (Low $=231.7 \mathrm{~ms}$, Medium $=224.1 \mathrm{~ms}$, High $=222.4$ ), showed a parietal maxima, with a right $>$ left effect also reaching significance (see Table 2 for effect summaries and means). Across the scalp, the P2 showed a Go > Nogo effect. On the Lateral dimension, both the right > left and midline > hemispheres effect was larger for the Go than Nogo stimuli, highlighting an enhanced Go relative to the Nogo $\mathrm{P} 2$ in the right hemisphere.

Globally, the P2 component was the largest in the Low condition and decreased linearly with increasing time pressure (i.e. L > M > H). Importantly, between stimuli (i.e. Go vs. Nogo), 
the Low condition showed a small Nogo > Go effect, while the Medium and High conditions displayed the opposite pattern - highlighting a reduction in the Nogo P2 with increasing task difficulty (see Figure 5). This effect was most apparent in posterior regions, with the Low condition showing a larger Posterior > Frontal effect for Nogo compared to Go (parietal vs. frontal difference: Nogo 3.5 vs. Go $2.7 \mu \mathrm{V}$ ), which was relatively equipotential for the Medium (Nogo 3.1 vs. Go $3.2 \mu \mathrm{V}$ ), and reversed for the High condition (Nogo 1.7 vs. Go $4.2 \mu \mathrm{V}$; see Figure 6 top panel).

Table 2. Significant results for the early ERP components, the N1 and P2.

\begin{tabular}{|c|c|c|c|c|c|}
\hline Measure & Effect & Contrast & Details & $\mathbf{F}$ & $\eta^{2}$ \\
\hline \multirow[t]{13}{*}{$\mathrm{N} 1$} & $\mathrm{~S}$ & $f$ vs. $p$ & -1.7 vs. 0.0 & $21.08^{\star \star *}$ & .243 \\
\hline & & c vs. $f / p$ & -1.2 vs. -0.9 & $12.72^{* *}$ & .012 \\
\hline & L & I vs. r & -1.0 vs. -0.8 & $4.61^{*}$ & .039 \\
\hline & & m vs. $I / r$ & -1.1 vs. -0.9 & $5.86^{*}$ & .039 \\
\hline & S $\times$ Cond & Czvs. Fz/pz & Low: -1.1 vs. -0.4 & & \\
\hline & & & Med: -1.1 vs. -1.2 & & \\
\hline & & & High: -1.4 vs -1.0 & $4.48^{*}$ & .009 \\
\hline & $L \times$ Cond & $m$ vs. $I / r$ & Low: -1.0 vs. -0.5 & & \\
\hline & & & Med: -1.2 vs. -1.1 & & \\
\hline & & & High: -1.1 vs. -1.1 & $5.14^{* *}$ & .063 \\
\hline & Stim $\times$ Cond & Go vs. Nogo & Low: -1.1 . vs. -0.2 & & \\
\hline & & & Med: -1.2 vs. -1.1 & & \\
\hline & & & High: -0.6 vs. -1.7 & $6.55^{\star *}$ & .187 \\
\hline \multirow[t]{14}{*}{ P2 } & $S$ & fvs.p & 2.3 vs. 5.4 & $51.47^{* \star *}$ & .430 \\
\hline & L & I vs. r & 3.4 vs. 4.3 & $27.89^{* \star *}$ & .193 \\
\hline & Stim & Go vs. Nogo & 4.6 vs. 3.4 & $12.58^{\star \star}$ & .146 \\
\hline & L x Stim & I vs. r & Go: 3.8 vs. 5.1 & & \\
\hline & & & Nogo: 3.1 vs. 3.6 & $16.88^{* \star *}$ & .127 \\
\hline & & $m$ vs. $I / r$ & Go: 4.8 vs. 4.4 & & \\
\hline & & & Nogo: 3.4 vs. 3.3 & $5.60^{*}$ & .030 \\
\hline & Cond & Low vs. High & 3.3 vs. 5.4 & $5.29^{*}$ & .085 \\
\hline & Stim $\times$ Cond & Go vs. Nogo & Low: 5.1 vs. 5.8 & & \\
\hline & & & Med: 4.1 vs. 2.3 & & \\
\hline & & & High: 4.5 vs. 2.1 & $8.34^{* *}$ & .193 \\
\hline & S $\times$ Stim $\times$ Cond & fvs.p & Low: Go, 3.5 to 6.2 ; Nogo, 3.7 to 7.2 & & \\
\hline & & & Med: Go, 2.3 to 5.5 ; Nogo, 0.7 to 3.8 & & \\
\hline & & & High: Go, 2.5 to $6.7 ;$ Nogo, 1.3 to 3.0 & $4.89^{*}$ & .128 \\
\hline
\end{tabular}

Details column represents mean amplitude in $\mu \mathrm{V}$. Abbreviations for this and subsequent tables in this study: Cond, Condition: Low/Medium/High task difficulty. Low, Low task difficulty condition. Med, Medium difficulty condition, High, High difficulty condition. Stim, Stimulus type: Go/NoGo. Lateral (L) abbreviations: I, mean left hemisphere (F3, C3, P3); r, mean right hemisphere (F4, C4, P4); I/r, mean of the left and right hemispheres (F3, C3, P3, F4, C4, P4); m, mean of the midline (Fz, Cz, Pz). Sagittal (S) abbreviations: $f$, mean frontal (F3, Fz, F4); $p$, mean parietal (P3, Pz, P4); $c$, mean central $(C 3, C z, C 4) ; f / p$, mean of frontal and parietal (F3, Fz, F4, P3, Pz, P4). Lateral by Sagittal (L x S) interactions: sites (e.g. f4) represent position on scalp (for e.g. frontal right hemisphere); f3/p3, mean of frontal and parietal left hemisphere; f4/p4, mean of frontal and parietal right hemisphere; $\mathrm{fz} / \mathrm{pz}$, mean of frontal and parietal midline; f3/f4, mean of frontal left and right hemispheres; $\mathrm{p} 3 / \mathrm{p} 4$, mean of parietal left and right hemispheres; $c 3 / c 4$, mean of central left and right hemispheres; $\mathrm{f} 3 \mathrm{f} 4 / \mathrm{p} 3 \mathrm{p} 4$, mean of frontal and parietal left and right hemispheres. 


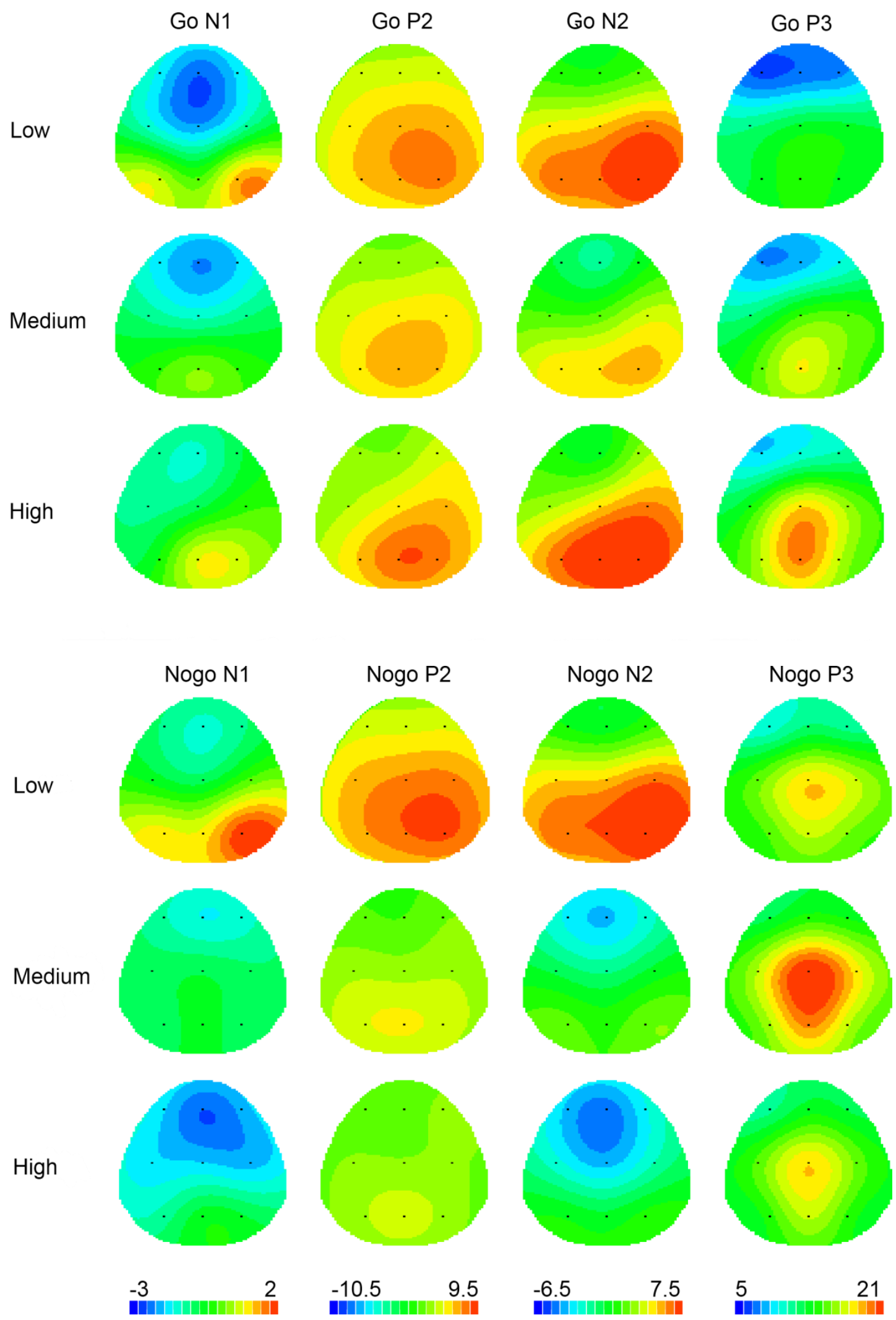

Figure 4. Topographic maps for each ERP component to Go (top panel) and Nogo (bottom panel) stimuli separately. Scale values represent the ends of the colour scale in $\mu \mathrm{V}$ for each component. Darkest blue $=$ negativity, red $=$ positivity. 
In summary, the analyses of the early ERP potentials to Go/Nogo stimuli showed increased Nogo N1 amplitudes across the scalp with increasing task difficulty. However, the Nogo P2 declined with time pressure, showing the smallest amplitudes over posterior regions in the High condition.

\subsubsection{N2}

N2 (mean latency $272.9 \mathrm{~ms}$ ) peaked earlier for Go $(269.8 \mathrm{~ms})$ than Nogo stimuli $(276.1 \mathrm{~ms}$; $F=5.15, p=.007, \eta 2=.085)$, and decreased linearly with task difficulty, being shorter for the High (265.6 ms), than Medium (270.5 ms) and Low conditions (282.7 ms; $F=10.24, p$ $=.002, \eta 2=.152)$,

The N2 showed a frontal maximum, and was larger in the left than right hemisphere, and greatest in the midline (see Table 3). N2 amplitude was larger to Nogo than Go stimuli, with the left > right effect being greater for the Go than Nogo N2, due mainly to an enhanced midline $>$ hemispheres effect for the Nogo N2.

Table 3. Significant results for the N2.

\begin{tabular}{|c|c|c|c|c|c|}
\hline Measure & Effect & Contrast & Details & $\mathbf{F}$ & $\eta^{2}$ \\
\hline \multirow[t]{19}{*}{ N2 } & S & fvs.p & -0.8 vs. 4.6 & $158.43^{\star \star *}$ & .687 \\
\hline & L & Ivs.r & 1.6 to 2.8 & $46.49^{* * *}$ & .242 \\
\hline & & $\mathrm{m}$ vs. $\mathrm{l} / \mathrm{r}$ & 1.6 vs. 2.2 & $16.98^{* * *}$ & .102 \\
\hline & Stim & Go vs. Nogo & 3.5 vs. 0.4 & $46.86^{\star \star *}$ & .343 \\
\hline & L x Stim & Ivs.r & Go: 2.9 vs. 4.5 & & \\
\hline & & & Nogo: 0.3 vs. 1.1 & $14.10^{* * *}$ & .111 \\
\hline & & $m$ vs. $1 / r$ & Go: 3.3 vs. 3.7 & & \\
\hline & & & Nogo: -0.2 vs. 0.7 & $19.43^{\star \star \star}$ & .079 \\
\hline & Cond & Low vs. High & 4.1 vs. 1.1 & $9.22^{\star *}$ & .139 \\
\hline & & Med vs. High/Low & 0.9 vs. 2.6 & $3.97^{\star}$ & .065 \\
\hline & Stim $\times$ Cond & Go vs. Nogo & Low: 4.0 vs. 4.1 & & \\
\hline & & & Med: 2.5 vs. -0.8 & & \\
\hline & & & High: 4.2 vs. -2.0 & $16.22^{\star \star \star}$ & .238 \\
\hline & S $\times$ Stim $\times$ Cond & fvs.p & Low: Go, 0.9 to 6.5 ; Nogo, 0.6 to 6.8 & & \\
\hline & & & Med: Go, -0.4 to $5.2 ;$ Nogo, -3.3 to 1.6 & & \\
\hline & & & High: Go, 1.1 to $7.2 ;$ Nogo, -3.9 to 0.4 & $3.25^{*}$ & .075 \\
\hline & & c vs. f/p & Low: Go, 4.7 to $3.7 ;$ Nogo, 5.1 to 3.7 & & \\
\hline & & & Med: Go, 2.7 to $2.4 ;$ Nogo, -0.7 to -0.9 & & \\
\hline & & & High: Go, 4.3 to $4.2 ;$ Nogo, -2.8 to -1.8 & $6.81^{* *}$ & .043 \\
\hline
\end{tabular}



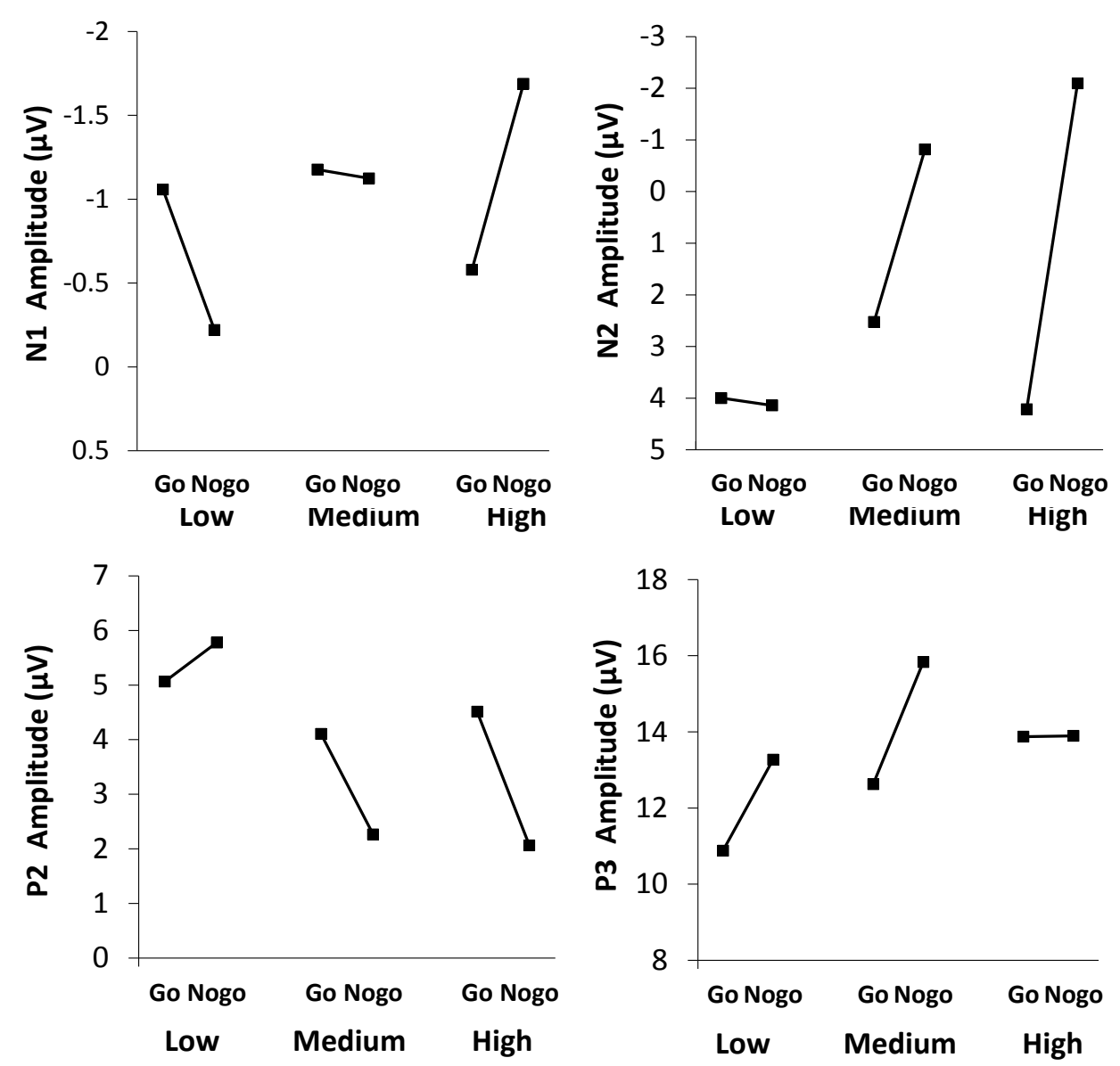

Figure 5. Go vs. Nogo amplitude across the scalp, by task difficulty condition, for the N1 (top left panel), P2 (top right panel), N2 (bottom left) and P3 (bottom right panel). 
Linear and quadratic interactions revealed that $\mathrm{N} 2$ amplitude (i.e. Go + Nogo) increased with increasing task difficulty (i.e. $\mathrm{H}>\mathrm{M}>\mathrm{L}$ ), which was characterised by a rapid rise from Low to Medium, but a relatively equipotential component for the Medium/High conditions. Notably, the Nogo > Go effect increased linearly with task difficulty (i.e. $H>M>L$ ), highlighting an augmented Nogo N2 across the scalp particularly for the High condition (see Figure 5). As shown in Figure 6, the High condition displayed an enhanced Nogo > Go N2 effect in parietocentral regions compared to the Medium/Low conditions. This is evidenced by a reduced frontal > parietal gradient (parietal vs. frontal difference: Nogo 4.3, Go $6.1 \mu \mathrm{V}$ ) and an increased central > frontal/parietal effect (central vs. frontal/parietal difference: Nogo 1.0, Go $0.1 \mu \mathrm{V}$ ) to Nogo compared to Go stimuli for the High condition, an effect which was reduced in the Medium (P vs. F diff.: Nogo 4.9, Go $5.6 \mu \mathrm{V}$; c vs. f/p: Nogo, 0.3, Go 0.3 $\mu \mathrm{V}$ ) and relatively equipotential for the Low condition (P vs. F diff.: Nogo 5.8, Go $5.6 \mu \mathrm{V}$; c vs. f/p: Nogo, 1.4, Go $1.0 \mu \mathrm{V})$. The association between inhibition performance and the Nogo > Go N2 effect was evaluated by calculating Pearson's correlation between Nogo errors and the N2 effect (Nogo N2 - Go N2 at Fz, with larger negative scores indicating a larger Nogo > Go N2 effect). Results indicated an association between poorer inhibitory performance and larger Nogo N2 amplitudes $(r=-.41, p=.001)$.

\subsubsection{P3}

P3 (mean latency $381.6 \mathrm{~ms}$ ) peaked later for Nogo (401.8 ms) than Go stimuli (373.4 ms; $F$ $=42.56, p<.001, \eta 2=.372$ ). This effect differed between conditions: with the $\mathrm{P} 3$ peaking much later for Nogo than Go stimuli for the High (Go vs. Nogo difference: $52 \mathrm{~ms}$ ) than the Medium (Go vs. Nogo difference: $15 \mathrm{~ms}$ ) and Low conditions (Go vs. Nogo difference: 17 $\mathrm{ms} ; F=7.41, p=.001, \eta 2=.130)$.

The P3 showed parietocentral and right midline maxima (see Table 4) in the Sagittal and Lateral dimensions, respectively. P3 amplitude was globally larger to Nogo than Go stimuli. A reduced parietal > frontal gradient (parietal vs. frontal difference: Nogo 3.6, Go $7.1 \mu \mathrm{V}$ ) and an increased central > frontal/parietal effect in Nogo compared to Go stimuli (central vs. frontal/parietal difference: Nogo 2.8, Go $1.8 \mu \mathrm{V}$ ), highlighted a more anterior P3 to Nogo relative to Go stimuli. In addition, while the right > left effect was reduced for Nogo relative to Go stimuli, the midline hemisphere effect was increased. 
Table 4. Significant results for the P3.

\begin{tabular}{|c|c|c|c|c|c|}
\hline Measure & Effect & Contrast & Details & $\mathbf{F}$ & $\eta^{2}$ \\
\hline \multirow[t]{25}{*}{ P3 } & S & fvs.p & 9.8 vs. 15.1 & $121.23^{* \star *}$ & .792 \\
\hline & & c vs. $f / p$ & 14.8 vs. 12.5 & $113.89^{* \star *}$ & .202 \\
\hline & L & Ivs.r & 12.1 vs. 13.2 & $26.71^{* * *}$ & 117 \\
\hline & & m vs. $1 / r$ & 14.4 vs. 12.7 & $99.73^{* * *}$ & .381 \\
\hline & Stim & Go vs. Nogo & 12.1 vs. 14.3 & $13.57^{\star \star}$ & .176 \\
\hline & S x Stim & fvs.p & Go: 8.0 vs. 15.1 & & \\
\hline & & & Nogo: 11.6 vs. 15.2 & $54.34^{* * *}$ & .376 \\
\hline & & c vs. f/p & Go: 13.3 vs. 11.5 & & \\
\hline & & & Nogo: 16.2 vs. 13.4 & $24.33^{* * *}$ & .049 \\
\hline & L x Stim & Ivs. $r$ & Go: 11.0 vs. 12.4 & & \\
\hline & & & Nogo: 13.2 vs. 14.0 & $5.24^{*}$ & .038 \\
\hline & & $\mathrm{m}$ vs. $\mathrm{l} / \mathrm{r}$ & Go: 13.0 vs. 11.7 & & \\
\hline & & & Nogo: 15.8 vs. 13.6 & $28.07^{\star \star \star}$ & .141 \\
\hline & Stim $\times$ Cond & Go vs. Nogo & Low: 10.8 vs. 13.3 & & \\
\hline & & & Med: 12.6 to 15.8 & & \\
\hline & & & High: 13.9 vs. 13.9 & $3.34^{*}$ & .086 \\
\hline & S x Stim X Cond & fvs. $p$ & Low: Go, 6.3 to $12.2 ;$ Nogo, 10.3 to 14.2 & & \\
\hline & & & Med: Go, 8.4 to $16.0 ;$ Nogo, 12.6 to 16.9 & & \\
\hline & & & High: Go, 9.4 to $17.0 ;$ Nogo, 11.8 to 14.4 & $3.35^{\star}$ & .046 \\
\hline & & c vs. f/p & Low: Go, 11.1 to 9.3 ; Nogo, 15.3 to 12.3 & & \\
\hline & & & Med: Go, 13.5 to $12.2 ;$ Nogo, 18.0 to 14.8 & & \\
\hline & & & High: Go, 15.3 to 13.2 ; Nogo, 15.5 to 13.1 & $4.41^{*}$ & .018 \\
\hline & L x Stim X Cond & $\mathrm{m}$ vs. $\mathrm{l} / \mathrm{r}$ & Low: Go, 10.2 to $9.7 ;$ Nogo, 14.3 to 12.8 & & \\
\hline & & & Med: Go, 13.3 to $12.3 ;$ Nogo, 17.6 to 14.9 & & \\
\hline & & & High: 15.3 to $13.2:$ Nogo, 15.4 to 13.1 & $6.84^{* *}$ & .069 \\
\hline
\end{tabular}

$*=<.05, * *=<.01, * * *=<.001$

Globally, the Nogo > Go P3 effect increased from the Low (Go vs. Nogo difference: $2.5 \mu \mathrm{V}$ ) to the Medium condition (Go vs. Nogo difference: $3.2 \mu \mathrm{V}$ ), contrasting with the High, which showed little difference between stimulus types (Go vs. Nogo difference: $0.0 \mu \mathrm{V}$; Figure 5). The distribution of the Nogo > Go P3 effect also differed between conditions: the Nogo relative to the Go P3 showed a more anterior focus for the Medium (parietal vs. frontal difference: Nogo 4.2, Go $7.6 \mu \mathrm{V}$; central vs. frontal/parietal difference: Nogo 3.2, Go 1.3 $\mu \mathrm{V}$ ) than the Low condition (parietal vs. frontal difference: Nogo 4.2, Go $6.0 \mu \mathrm{V}$; central vs. frontal/parietal difference: Nogo 1.5, Go $3.0 \mu \mathrm{V}$ ), with this effect being reduced for the High condition (parietal vs. frontal difference: Nogo 2.6, Go $7.6 \mu \mathrm{V}$; central vs. frontal/parietal difference: Nogo 2.2, Go $2.1 \mu \mathrm{V}$ ). This effect highlights a reduction in centroparietal Nogo P3 activity for the High condition (see Figure 6). Similarly, on the Lateral dimension, a midline > hemispheres effect for Nogo relative to Go stimuli increased slightly from the Low (Mid. vs. Hem. diff.: Nogo 1.5, Go $0.5 \mu \mathrm{V}$ ) to the Medium condition (Mid. vs. Hem. diff.: Nogo 2.7, Go $1.0 \mu \mathrm{V}$ ), but was reduced for the High (Mid. vs. Hem. diff.: Nogo 2.3, Go $2.1 \mu \mathrm{V})$. 

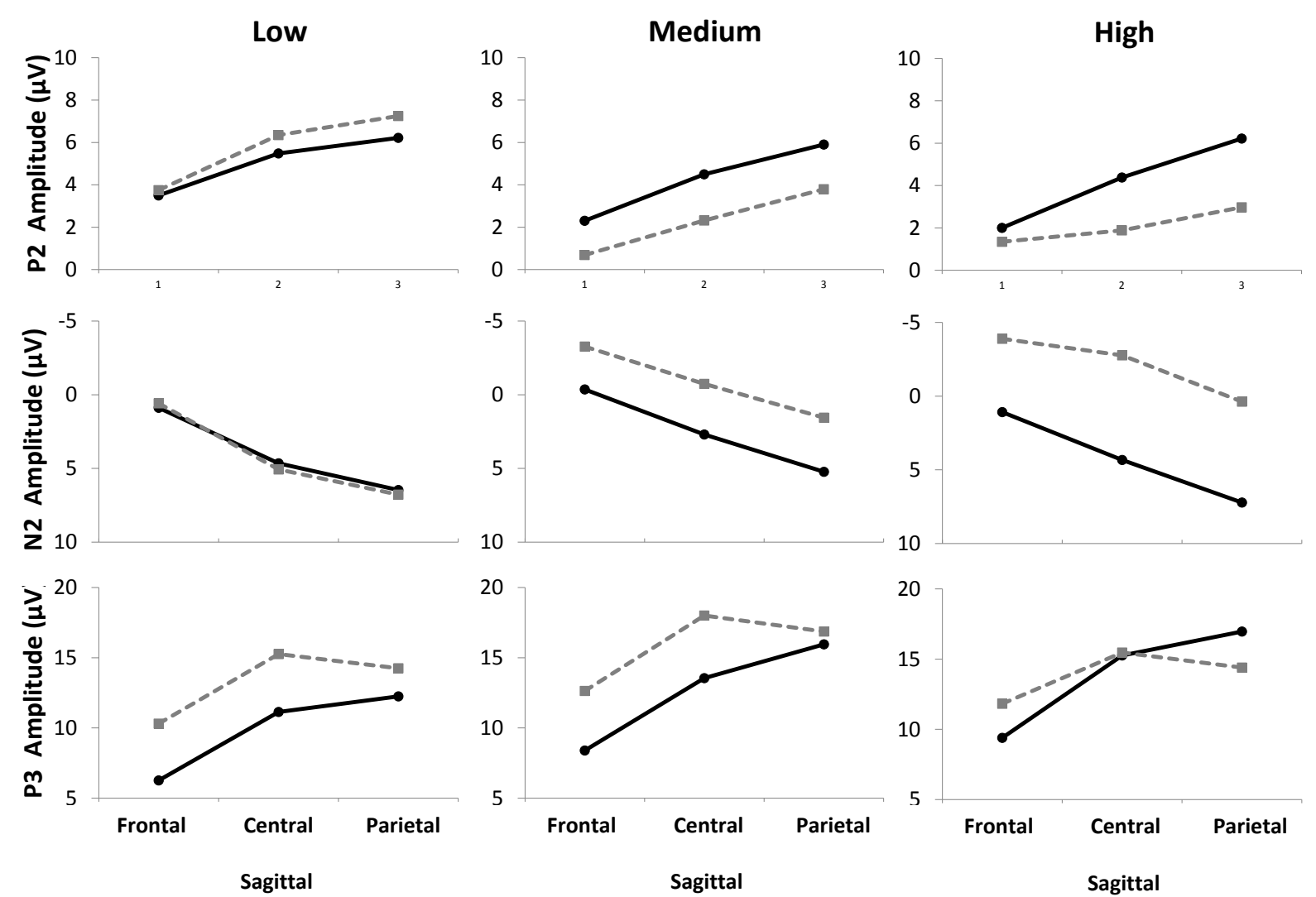

$\rightarrow-G o-+N o g o$

Figure 6. The Stimulus $x$ Sagittal x Condition interactions for P2 (top panel), N2 (middle panel) and P3 amplitude (bottom panel). Note: Frontal= mean of F3, Fz, F4; Central = mean of C3, Cz, C4; Parietal = mean of P3, Pz, P4. 
In summary, the Nogo > Go N2 effect increased incrementally and peaked earlier as a function of task difficulty, with the largest amplitudes and shortest latencies in the High condition. By contrast, while the Nogo > Go P3 effect increased from Low to the Medium condition, it was significantly reduced for the High condition. Differences in the distribution for the Nogo > Go P3 effect were most apparent frontocentrally between the Low and Medium conditions, while the High showed a reduction in Nogo P3 activity in the centroparietal region.

\section{Discussion}

The primary aim of this study was to examine the influence of varying task difficulty, by the use of reaction time deadline, on the behavioural and ERP indices of inhibitory control during performance of the Go/Nogo task. In addition, we investigated whether the effect of task difficulty would also extend to the early ERP potentials, task-related arousal and perceived effort.

\subsection{Task Performance}

Our results indicate that task performance was significantly affected by variations in task difficulty. Specifically, Go and Nogo errors incrementally increased with each increase in task difficulty (i.e. RTD reduction: Table 1), with the greatest number of errors in the High condition. Importantly, modulations in task difficulty were also reflected by concurrent increases in perceived effort (Figure 2), consistent with the idea that greater effortful control is required when the need to inhibit is high (Jodo and Kayama, 1992). Since previous research has either not utilised graded task difficulty levels (for e.g. Band et al., 2003; Smith et al., 2006), or did not report task performance data (Jodo and Kayama, 1992), these results provide clear self-report and behavioural evidence that Go/Nogo task difficulty can be incrementally increased by the use of RTDs. 


\subsection{SCL Arousal}

Arousal level did not differ among conditions and did not appear to be related to task difficulty or performance in the present study. Combined with the findings of cumulative increases in Go/Nogo errors with increasing task difficulty, this SCL result differs from previous work suggesting that arousal is dependent on the difficulty level of a given task (Yerkes and Dodson, 1908). It is interesting to note, however, that arousal level was not completely static among conditions, with a tendency for arousal to show a Low/High > Medium effect - in line with previous work suggesting increased arousal levels during slow/fast, relative to medium speeds of presentation (Sanders, 1983). Alternatively, a more complete explanation might be in regard to the use of skin conductance level as a measure of arousal in the current research. In a series of studies, Barry and colleagues (e.g. Barry et al., 2005) experimentally differentiated between 'arousal', referring to the current energetic state of an individual, and 'activation', which refers to the task-related mobilisation of arousal. Notably, arousal was not found to be related to any of the performance variables, but instead, task-related activation significantly determined improvements in both reaction time and errors. Recent work by this group has also reported the classic inverted-U relationships between task-related activation and performance in a variety of tasks (VaezMousavi and Osanlu, 2008; VaezMousavi et al., 2009). Thus, it might be advantageous in future research to employ measures of task-related activation to more thoroughly explore the influence of task difficulty on arousal/activation.

\subsection{Early ERP Findings}

Although the primary aim of this study was to investigate the influence of a task difficulty manipulation on the inhibition-related ERP components of the N2 and P3, we report significant condition effects for the early exogenous potentials of the N1 and P2. Specifically, while the Low condition showed a Go > Nogo N1 effect across the scalp, this effect was reversed and increased with task difficulty, to show a large Nogo > Go effect for the High condition (see Figure 4 for head maps and Figure 5 for Go vs. Nogo plots). Previous examinations linking N1 and RT have produced mixed results: Bahramali, Gordon, \& Li (1998) and Karlin et al., (1971) reported a larger N1 with fast responses, while Starr, Sandroni, and Michalewski (1995) found no significant differences. The N1 component is 
generally thought to represent the initial sensory extraction of, and attention to stimuli (Näätänen \& Picton, 1987), while previous investigators have interpreted an increased N140 to NoGo stimuli as reflecting an early manifestation of inhibitory processing (Nakata et al., 2004). Therefore, an enhanced Nogo N1 may reflect the greater visual resources required for inhibitory processing as a function of task difficulty - potentially indicating that the early extraction of stimulus information can be modulated by task demands (Miller et al., 2011), with implications for information processing at later stages (Smith et al., 2004).

While typically considered an exogenous component, the functional significance of the P2 in Go/Nogo tasks has yet to be resolved (Benikos and Johnstone, 2009; Wiersema et al., 2006). In discrimination paradigms, the $\mathrm{P} 2$ is thought to be involved in the protection against interference from irrelevant stimuli (Garcia-Larrea et al., 1992), giving the imperative stimulus a clear path for further processing (Oades, 1998). Ross and Tremblay (2009) posit that enhanced parietal P2 amplitudes reflects the physiological processes associated with improved task performance - an interpretation in line with reports of larger P2s with concurrent reductions in reaction time (Johnstone et al., 2005; Tonga et al., 2009) and commission errors (Johnstone et al., 2005; Kenemans et al., 1993; Smid et al., 1999). In line with this, the Low condition showed a slightly larger Nogo than Go P2; in contrast to the Medium and High conditions, which displayed a large reduction in Nogo P2 amplitude primarily in posterior regions. Since larger P2s have also been linked to deliberately initiated actions (Kühn et al., 2009), it is possible that with sufficient time to respond, participants in the Low condition were more able to appropriately respond to Go/Nogo stimuli. In contrast, despite the enhanced activation of the Nogo N1, increased task difficulty in the High conditions could have reduced the ability of these participants to suppress extraneous stimuli and inhibit responses. These results are consistent with previous research suggesting that although the primary emphasis in the response inhibition literature has been the N2/P3 complex, earlier waveform components such as the N1 and P2 may play an important role in inhibition success (Roche et al., 2005; Thomas et al., 2009). It thus seems reasonable to suggest that the Nogo P2 reductions seen in this study are largely due to task difficulty effects, and could be linked, in part, to impairments in inhibitory processing and declines in performance. 


\subsection{Inhibition-related ERP components}

Across conditions, we replicated the well-known inhibition-related effects of increased N2 amplitudes and a more anterior P3 to Nogo relative to Go stimuli (Eimer, 1993; Kenemans et al., 1993; Oddy et al., 2005). Go N2 peaked earlier than the Nogo N2 (Jodo and Kayama, 1992), while the reverse was found for the P3 (i.e. Nogo P3 > Go P3 latency; Fallgatter and Strik, 1991; Salisbury et al., 2004). Finally, the current study also reports globally enhanced $\mathrm{N} 2$ amplitudes with increasing task difficulty, in line with previous research linking larger N2 peaks with faster responses (Bahramali et al., 1998; Starr et al., 1995).

\section{$4.4 \mathrm{~N} 2$}

The Nogo > Go N2 effect was larger (Figure 5) and occurred earlier with each increase in task difficulty, as has been reported in previous studies (Band et al., 2003; Falkenstein, 2006; Jodo and Inoue, 1990). Since previous research by Jodo \& Kayama (1992) did not report behavioural data, this study demonstrates that graded increases in task difficulty (via RTD) are reflected by incremental amplitude increases and reductions in Nogo N2 latency. In a frequently cited study, Falkenstein et al. (1999) reported that the Nogo N2 was larger and earlier in good compared to poor inhibitors (as measured by the number of commission errors), interpreted as due to a stronger and earlier inhibition process by the good inhibitors. In contrast, the present study reports the opposite effect (i.e. shorter latencies and increased Nogo N2 amplitudes) for the high difficulty condition, which showed the greatest number of inhibition errors. Given the significant correlation indicating an inverse relationship between Nogo N2 amplitude and inhibition performance, this argues against the interpretation of the Nogo N2 as pre-motor index of inhibitory control (e.g. Kok, 1999). Recently, however, evidence linking the N2 to response conflict has been accumulating (Smith, Johnstone \& BarrySmith et al., 2010). The conflict theory of N2 predicts increased competition between Go and Nogo representations on correct trials when participants are required to emphasise speed over accuracy (van Veen and Carter, 2002). Thus, it might be that variations in the amplitude $\mathrm{N} 2$ reflect incremental increases in response conflict with task difficulty, rather than inhibitory control. 
It is noteworthy to report that the Nogo N2 also appeared to change its distribution with enhanced difficulty, displaying an increased Nogo > Go N2 effect at centroparietal regions for the High condition (Figure 6). A prominent review of the N2 has suggested that it does not reflect a single underlying process, but rather a family of sub-components related to cognitive control (Folstein and Van Petten, 2008). In line with this, it may be that this Condition $x$ Site interaction is suggestive of different neural generators of the N2 for each condition (Johnson, 1993). According to Kok (2001), changes in cognitive processing are a common effect of task difficulty manipulations. Therefore, it may be that different neural generators of the $\mathrm{N} 2$ are differentially sensitive to task difficulty in the Go/Nogo task, potentially leading to alterations in its distribution.

\section{$4.5 \mathrm{P3}$}

The Nogo > Go P3 effect increased from the Low to the Medium condition, with little difference found between the stimulus types for the High condition. A more anterior NoGo than Go P3 is considered to be reflective of inhibitory processing by some researchers (Bekker et al., 2005; Kok et al., 2004; Smith and Douglas, 2011), and via the use of three task difficulty levels, the results from the present study appear to support this idea. That is, the larger Nogo than Go P3 for the Medium than Low condition (primarily at frontocentral regions) may be reflective of an increased requirement for inhibitory processing with increasing task difficulty. Beyond this point, however, task difficulty seems to overwhelm the response inhibition mechanism, leading to reductions in the Nogo P3 effect. Indeed the findings of longer Nogo P3 latency and 25\% commission errors for the High as opposed to $11.1 \%$ commission errors for the Medium condition, is consistent with this interpretation. Studies investigating workload (for a review see Kok, 1997) and semantic categorisation (Maguire et al., 2009; Maguire et al., 2011) have reported similar reductions in P3 amplitude with increasing task difficulty

However, it is interesting to note that the distribution of the Nogo P3 revealed amplitude reductions for the High condition at centroparietal regions (see Figure 6). Thus it may argued that the relative decline of the Nogo P3 during high task difficulty may not be solely due to variations in inhibitory processing given that, (a) it is not a frontal change, (b) frontal Nogo P3 amplitude does not appear to differ substantially between the Medium and High 
conditions (Figure 6), and (c) previous research has shown a clear relationship between frontal lobe activation and inhibitory processing (e.g. Rubia et al., 2001). Reduced Nogo P3 amplitudes over centroparietal regions with increasing task difficulty may thus be better explained in terms of a decrease in the ability to evaluate inhibition success (e.g. Beste et al., 2010). That is, although ISIs were kept consistent between conditions, participants in the High condition may have perceived that too little time was available to adequately monitor the inhibition outcome, leading to reductions in the centroparietal Nogo P3. It can also be argued that the functional interpretation of the Nogo P3 is dependent on the scalp topography (Tekok-Kilic et al., 2001; Vallesi, 2011), and that two distinct processes are contributing to the differences between conditions: a response inhibition process which produces the more anterior Nogo than Go P3for the Low and Medium conditions, and an inhibition monitoring process that is reflected by the centroparietal reductions for the High condition. However, this notion requires further investigation.

This investigation is not without limitations. Future studies could consider the use of a within-subjects design, which would add statistical power and reduce the error variance between conditions. In addition, due to the use of a psychology undergraduate population, all three task difficulty conditions contained many more females than males. While the issue of gender effects has not been well-studied in the Go/Nogo context, recent research by Yuan and colleagues (2008) has reported that women showed shorter latencies and larger amplitudes for deviant-related P2, N2 and P3 components. Accordingly, the use equal number of males and females might be useful in future research to further clarify the effect of task difficulty on inhibitory performance and processing.

\subsection{Conclusions}

In summary, this study reports that task difficulty in the Go/Nogo task can be effectively manipulated by varying RTDs. In the context of declines in task performance and the absence of arousal effects, incremental amplitude increases and reductions in latency were seen for the Nogo N2, potentially indicating enhanced response conflict with greater Go/Nogo task demands. In contrast, the NogoP3 effect was reduced with increasing task difficulty, suggesting that reductions in RTD may serve to impair inhibition-related processing or monitoring. Finally, our data also imply that the inhibitory control may not be 
solely manifested by modulations in the $\mathrm{N} 2$ and $\mathrm{P} 3$, but that differential processing of the $\mathrm{N} 1$ and the P2 may also influence Go/Nogo task performance. These findings have real-world significance in light of a growing body of literature examining techniques for training inhibitory control as a way to ameliorate inhibitory control deficits seen in disorders such as ADHD. Importantly, mixed results in this line of research have been suggested to be partly due to a lack of optimal task difficulty manipulation. Thus, taken together, this study provides useful baseline behavioural and ERP data for appropriately manipulating task difficulty in Go/Nogo tasks, and potentially offers a constructive avenue for researchers attempting to design effective inhibition training paradigms. 


\section{References}

Aron, A.R., Robbins, T.W., Poldrack, R.A., 2004. Inhibition and the right inferior frontal cortex. Trends in Cognitive Sciences $8170-177$.

Bahramali, H., Gordon, E., Li, W.M., 1998. Fast and slow reaction time changes reflected in ERP brain function. Int. J. Neurosci. 93, 75-85.

Band, G.P., Ridderinkhof, K.R., van der Molen, M.W., 2003. Speed-accuracy modulation in case of conflict: The roles of activation and inhibition. Psychol. Res. 67, 266-279.

Band, G.P., van Boxtel, G.J., 1999. Inhibitory motor control in stop paradigms: Review and reinterpretation of neural mechanisms. Acta Psycholgica 101, $179-211$.

Barry, R.J., Clarke, A.R., McCarthy, R., Selikowitz, M., Rushby, J.A., 2005. Arousal and Activation in a Continuous Performance Task: An Exploration of State Effects in Normal Children. Journal of Psychophysiology 19, 91-99.

Barry, R.J., Johnstone, S.J., Clarke, A.R., Rushby, J.A., Brown, C.R., McKensie, D.N., 2007. Caffeine effects on ERPs and performance in an auditory Go/NoGo task. Clin. Neurophysiol. $118,2692-2699$.

Barry, R.J., Sokolov, E.N., 1993. Habituation of phasic and tonic components of the orienting reflex. Journal of Psychophysiology 15, 39-42.

Bekker, E.M., Kenemans, J.L., Verbaten, M.N., 2005. Source analysis of the N2 in a cued Go/NoGo task. Cognitive Brain Research 22, 221 - 231.

Benikos, N., Johnstone, S.J., 2009. Arousal-state modulation in children with AD/HD. Clin. Neurophysiol. 120, 30-40.

Beste, C., Saft, C., Andrich, J., Gold, R., Falkenstein, M., 2008. Response inhibition in Huntington's disease - a study using ERPs and sLORETA. Neuropsychologia 46, 1290 1297.

Beste, C., Willemssen, R., Saft, C., Falkenstein, M., 2010. Response inhibition subprocesses and dopaminergic pathways: Basal ganglia disease effects. Neuropsychologi 48, 366-373.

Bradley, J.V., 1958. Complete counterbalancing of immediate sequential effects in a Latin square design. Journal of the American Statistical Association. 53, 525-528. 
Braver, T.S., Barch, D.M., Gray, J.R., Molfese, D.L., Snyder, A., 2001. Anterior cingulate cortex and response conflict: Effects if frequency, inhibition and errors. Cereb. Cortex 11, 825-836.

Bruin, K.J., Wijers, A.A., van Staveren, A.S., 2001. Response priming in a Go/Nogo task: Do we have to explain the Go/Nogo N2 effects in terms of response activation instead of inhibition? Clinical Neurophysiology 112, 660 - 1671.

Clark, J.M., 1996. Contributions of inhibitory mechanisms to unified theory in neuroscience and psychology. Brain Cogn. 30, $127-152$.

Donkers, F.C.L., van Boxtel, G.J.M., 2004. The N2 in go/no-go tasks reflects conflict monitoring not response inhibition. Brain Cogn. 56, 165 - 176.

Eimer, M., 1993. Effects of attention and stimulus probability on ERPs in a Go/Nogo task. Biol. Psychol. 35, 123-138.

Falkenstein, M., 2006. Inhibition, conflict and the Nogo-N2. Clin. Neurophysiol. 117, 1638 1640.

Falkenstein, M., Hoormann, J., Hohnsbein, J., 1999. ERP components in Go/Nogo tasks and their relation to inhibition. Acta Psychologica 101, 267-291.

Fallgatter, A.J., Strik, W.K., 1991. The NoGo-anteriorisation as a neurophysiological standard-index for cognitive response control. International Journal of Psychophysiology 32, $233-238$.

Folstein, J.R., Van Petten, C., 2008. Influence of cognitive control and mismatch on the N2 component of the ERP: A review. Psychophysiology 45, $152-170$.

Garcia-Larrea, L., Lukaszewicz, A.C., Mauguiere, F., 1992. Revisiting the oddball paradigm. Non-target vs. neutral stimuli and the evaluation of ERP attentional effects.

Neuropsychologia 30, $723-741$.

Howell, D.C., 2009. Statistical Methods for Psychology, 7th ed. Wadsworth Belmont, CA. Jasper, H.H., 1958. Report of the committee on methods of clinical examination in electroencephalography. Electroencephalography and Clinical Neurophysiology 10, 370375. 
Jodo, E., Inoue, K., 1990. Effects of practice on the P300 in a Go/NoGo task. EEG Clinica Neurophysiology 76, $249-257$.

Jodo, E., Kayama, Y., 1992. Relation of a negative ERP component to response inhibition in the Go/Nogo task. Electroencephalography and Clinical Neurophysiology 82, 477-482.

Johnson, J., R. , 1993. On the neural generators of the P300 component of the event-related potential. Psychophysiology 30, 90-97.

Johnstone, S.J., Pleffer, C.B., Barry, R.J., Clarke, A.R., Smith, J.L., 2005. Development of inhibitory processing during the Go/Nogo task. Journal of Psychophysiology 19, 11-23. Johnstone, S.J., Roodenrys, S., Phillips, E., Watt, A.J., Mantz, S., 2010. Combined working memory and inhibition training for children with AD/HD. ADHD Attention Deficit and Hyperactivity Disorders 2, 31-42.

Karlin, 1971. Auditory evoked potentials, motor potentials and reaction time.

Electroencephalogr. Clin. Neurophysiol. 31, 129-136.

Kenemans, J.L., Kok, A., Smulders, F.T., 1993. Event-related potentials to conjunctions of spatial frequency and orientation as a function of stimulus parameters and response requirements. Electroencephalogr. Clin. Neurophysiol. 88, 51-63.

Kok, A., 1986. Effects of degradation of visual stimuli on components of the event-related potential (ERP) in go/nogo reaction tasks. Biological Psychology 23, 21-38.

Kok, A., 1997. Event-related-potential (ERP) reflections of mental resourc s: a review and synthesis. Biological Psychology 45, 19-56.

Kok, A., 2001. On the utility of P3 amplitude as a measure of processing capacity. Psychophysiology 38, 557-577.

Kok, A., Ramautar, J.R., De Ruitera, M.B., Band, G.P.H., Ridderinkhof, K.R., 2004. ERP components associated with successful and unsuccessful stopping in a stop-signal task. Psychophysiology 41, 9-20.

Kühn, S., Gevers, W., Brass, M., 2009. The Neural Correlates of Intending Not to Do Something. Jounral of Neurophysiology 101(4):1913-20, 1913 - 1920.

Lindqvist, S., Thorell, L.B., 2009. A brief report: Manipulating task difficulty in laboratory measures of inhibitory control. Child Neuropsychology 15, 1-7. 
Maguire, M.J., Brier, M.R., Moore, P.S., Ferree, T.C., Ray, D., Mostofsky, S., Hart, J.J., Kraut, M.A., 2009. The influence of perceptual and semantic categorization on inhibitory processing as measured by the N2-P3 inhibitory responses. Brain Cogn. 71, 196-203. Maguire, M.J., White, J., Brier, M.R., 2011. How semantic categorization influences inhibitory processing in middle-childhood: An Event Related Potentials study. Brain Cogn. 76, $77-86$.

McCarthy, G., Wood, C.C., 1985. Scalp distributions of event-related potentials: an ambiguity associated with analysis of variance models. Electroencephalography and Clinical Neurophysiology 62, $203-208$.

Miller, M.W., Rietschel, J.C., McDonald, C.G., Hatfield, B.D., 2011. A novel approach to the physiological measurement of mental workload. Int. J. Psychophysiol. 80, 75-78.

Näätänen R, T., P., 1987. The N1 wave of the human electric and magnetic response to sound: a review and an analysis of the component structure. Psychophysiology $24,375-425$. Nakata, H., Inui, K., Nishihira, Y., Hatta, A., Sakamoto, M., Kida, T., Wasaka, T., Kakigi, R., 2004. Effects of go/nogo task event-related potentials following somatosensory stimulation. Clin. Neurophysiol. 115, 361-368.

Nieuwenhuis, S., Yeung, N., van den Wildenburg, W., Ridderinkhof, K.R., 2003.

Electrophysiological correlates of anterior cingulate function in a go/nogo task: effects of response conflict and trial type frequency. Cognitive and Affective Behavioral Neuroscience $3,17-26$.

Oades, R.D., 1998. Frontal, temporal and lateralized brain function in children with attentiondeficit hyperactivity disorder: a psychophysiological and

neuropsychological viewpoint on development. Behavior and Brain Research 94, 83 - 95. Oddy, B.W., Barry, R.J., Johnstone, S.J., Clarke, A.R., 2005. Removal of CNV effects from the N2 and P3 ERP components in a visual Go/NoGo task. Journal of Psychophysioly 19, $24-34$.

Picton, T.W., Bentin, S., Berg, P., Donchin, E., Hillyard, S.A., Johnson, R.J., Miller, G.A., Ritter, W., Ruchkin, D.S., Rugg, M.D., Taylor, M.J., 2000. Guidelines for using human event- 
related potentials to study cognition: Recording standards and publication criteria. Psychophysiology 37, 127-152.

Randall, W.M., Smith, J.L., in Press. Conflict and inhibition in the cued-Go/Nogo task. Clin. Neurophysiol.

Roche, R.A.P., Garavan, H., Foxe, J.J., O’ Mara, S.M., 2005. Individual differences discriminate event-related potentials but not performance during response inhibition. Experimental Brain Research 160, 60-70.

Ross, B., Tremblay, K.L., 2009. Stimulus experiencemodifies auditory neuromagnetic responses in young andolder listeners. Hear. Res. 248, 48 - 59.

Rubia, K., Russella, T., Overmeyera, S., Brammera, M.J., Bullmoreb, E.T., Sharmaa, T., Simmonsa, A., Williams, S.C.R., Giampietroa, V., Andrew, C.M., Taylor, E., 2001. Mapping motor inhibition: conjunctive brain activations across different versions of go/no-go and stop tasks. Neuroimage 13, 250-261.

Salisbury, D.F., Griggs, C.B., Shenton, M.E., McCarley, R.W., 2004. The Nogo P300 'anteriorisation effect' and response inhibition. Clin. Neurophysiol. 115, 1550-1558. Sanders, A.F., 1983. Towards a model of stress and performance. Acta Psychologica 53, $61-97$.

Semlitsch, H.V., Anderer, P., Schuster, P., Presslich, O.A., 1986. A solution for reliable and valid reduction of ocular artifacts, applied to the P300 ERP. Psychophysiology 23, $695-703$. Simmonds, D.J., Pekar, J.J., Mostofsky, S.H., 2008. Meta-analysis of Go/No-go tasks demonstrating that $\mathrm{fMRI}$ activation associated with response inhibition is task-dependent. Neuropsychologia 46, $224-232$.

Smid, H.G.O.M., Jakob, A., Heinze, H.J., 1999. An event-related brain potential study of visual selective attention to conjunctions of color and shape. Psychophysiology 36, 264 279.

Smith, J.L., Douglas, K.M., 2011. On the use of event-related potentials to auditory stimuli in the Go/NoGo task. Psychiatry Research - Neuroimaging 193, 177-181.

Smith, J.L., Johnstone, S.J., Barry, R.J., 2004. Inhibitory processing during the Go/Nogo task: an ERP analysis of children with attention-deficit/hyperactivity disorder. Clinical Neurophysiology 115, $1320-1331$. 
Smith, J.L., Johnstone, S.J., Barry, R.J., 2006. Effects of pre-stimulus processing on subsequent events in a warned Go/NoGo paradigm: Response preparation, execution, and inhbition. International Journal of Psychophysiology 61, 121-133.

Smith, J.L., Johnstone, S.J., Barry, R.J., 2007. Response priming in the Go/NoGo task: the N2 reflects neither inhibition nor conflict. Clinical Neurophysiology 118, 343 - 355.

Smith, J.L., Johnstone, S.J., Barry, R.J., 2008. Movement-related potentials in the Go/NoGo task: the P3 reflects both cognitive and motor inhibition. Clinical Neurophysiology 119, 704 714

Smith, J.L., Smith, E., Provost, A.L., Heathcote, A., 2010. Sequence effects support the conflict theory of N2 and P3 in the Go/NoGo task. International Journal of Psychophysiology $75,217-226$.

Spencer, K.M., Dien, J., Donchin, E., 2001. Spatiotemporal analysis of the late ERP responses to deviant stimuli. Psychophysiology 38, 343-358.

Starr, A., Sandroni, P., Michalewski, H.J., 1995. Readiness to respond in a target detection task: pre- and post-stimulus event-related potentials in normal subjects. Electroencephalogr. Clin. Neurophysiol. 96, 76-92.

Tabachnick, B.G., Fidell, L.S., 1996. Using Multivariate Statistics. Harper-Collins, New York. Tekok-Kilic, A., Shucard, J.L., Shucard, D.W., 2001. Stimulus modality and Go/NoGo effects on P3 during parallel visual and auditory continuous performance tasks. Psychophysiology $38,578-589$.

Thomas, S.J., Gonsalvez, C.J., Johnstone, S.J., 2009. Sequence effects in the Go/NoGo task: Inhibition and facilitation. International Journal of Psychophysiology 74, 209-219.

Thorell, L.B., Lindqvist, S., Bergman-Nutley, S., Bohlin, G., Klingberg, T., 2009. Training and transfer effects of executive functions in preschool children. Developmental Science 12, $106-113$.

Tonga, Y., Robert, D., Melarab, A.R., 2009. P2 enhancement from auditory discrimination training is associated with improved reaction times. Brain Research $129780-88$. VaezMousavi, M., Osanlu, M., 2008. Skin Conductance Level Predicts Performance in a Balance Task. World Journal of Sport Sciences 4, 139-143. 
VaezMousavi, S.M., Barry, R.J., Clarke, A.R., 2009. Individual differences in task-related activation and performance. Physiol. Behav. 98, 326-330.

VaezMousavi, S.M., Barry, R.J., Rushby, J.A., Clarke, A.R., 2007. Evidence for differentiation of arousal and activation in normal adults. Acta Neurobiol. Exp. (Warsz). 67, 179-186.

Vallesi, A., 2011. Targets and non-targets in the aging brain: A go/nogo event-related potential study. Neurosci. Lett. 487, 313-317.

van Veen, V., Carter, C.S., 2002. The timing of action-monitoring processes in the anterior cingulate cortex. J. Cogn. Neurosci. 14, 593 - 602.

Wiersema, R., van Der Meere, J., Roeyers, H., Van Coster, R., Baeyens, D., 2006. Event rate and event-related potentials in ADHD. Journal of Child Psychology and Psychiatry 47, $560-567$.

Wodka, E.L., Simmonds, D.J., Mahone, E.M., Mostofsky, S.H., 2009. Moderate variability in stimulus presentation improves motor response control. Jounral of Clinical Experimental Neuropsychology 31, 483-488.

Yerkes, R.M., Dodson, J.D., 1908. The relation of strength of stimulus to rapidity of habitformation. Journal of Comparative Neurology 18, $459-482$.

Yuan, J., He, Y., Qinglin, Z., Chen, A., Li, H., 2008. Gender differeneces in behavioral inhibitory control: ERP evidence from a two-choice oddball task. Psychophysiology 45, 986993. 